\title{
Radiocarbon
}

1973

\section{BRATISLAVA RADIOCARBON MEASUREMENTS I}

\author{
S. USAC̆EV, P. POVINEC, M. CHUDY, and Š. ŠÁRÓ \\ Department of Nuclear Physics, Comenius University, \\ Bratislava, Czechoslovakia
}

\section{INTRODUCTION}

The Radiocarbon Dating Laboratory was established in 1967 at the Department of Nuclear Physics, Comenius University, as part of a program of low level counting research of the department and as a contribution to geophysical, geologic, archaeologic and hydrologic research.

The first series of $\mathrm{C}^{14}$ measurements were obtained using $\mathrm{CO}_{2}$ as a gas filling of a proportional counter (Povinec et al., 1968). Chemical and counting procedure adopted for radiocarbon dating of archaeologic and geologic samples has been described by Povinec et al. (1971). The counting unit is a $2.8 \mathrm{~L}$ copper proportional counter. For a two day count with 4 atm $\mathrm{CO}_{2}$ filling, the maximum measurable age is 37,000 years ( $4 \sigma$ criterion).

Recently we have adopted Lal's method of methane preparation, using $\mathrm{CO}_{2}$ and $\mathrm{H}_{2} \mathrm{O}$ in the presence of zinc dust and a ruthenium catalyst (Lal, 1965; Povinec, 1972).

For more precise measurements we have built an Oeschger-type proportional counter volume of $3.30 \mathrm{~L}$. At $5 \mathrm{~atm}$ of methane filling the maximum measurable age ( $48 \mathrm{hrs}$ counting, $4 \sigma$ criterion) is 50,000 years (Povinec et al., 1971). For samples of limited weight, a proportional counter volume of $0.4 \mathrm{~L}$ with plastic scintillator as an anticoincidence counter has been constructed (Provinec et al., 1971). $\mathrm{CO}_{2}$ is mostly used as the gas filling of this counter.

The numbers of pulses registered by scalers are printed out on a strip printer every 20 minutes. The data are processed on a computer, where age of samples and standard deviations are calculated. Calculations are based on the Lamont formulae (Broecker and Olson, 1961) and the standard deviations quoted $\left(1_{\sigma}\right)$ describe only the uncertainties associated with the sample, standard and background determinations. The contemporary reference used is $95 \%$ of the specific activity of NBS oxalic acid. Dates are calculated using the Libby half-life of $5568 \pm 30$ years with 1950 as the standard year of reference.

\section{ACKNOWLEDGMENTS}

The authors are indebted to D. Lal and D. P. Agrawal, Tata Institute of Fundamental Research, Bombay for technical instruction of one of us 
(P.P.) and for the supply of chemicals and intercalibration samples. We acknowledge cooperation of M. Šliga in construction of the preparation part of the equipment. Thanks are extended to N. Pišútová for processing data and to M. Oberhauserová, O. Košík, and V. Bankoš for technical assistance.

\section{SAMPLE DESCRIPTIONS}

I. INTERLABORATORY CHECK SAMPLES

To cross-check with other laboratories, we measured $\mathrm{C}^{14}$ concentration in a wood sample from the tomb of King Zoser and in charcoal samples, subm. by D. P. Agrawal.

TABle 1

Interlaboratory cross-check samples

\begin{tabular}{|c|c|c|c|c|}
\hline $\begin{array}{c}\text { Lab. } \\
\text { no. }\end{array}$ & Date & $\begin{array}{c}\text { Check } \\
\text { sample }\end{array}$ & Date & Reference \\
\hline Вa-79 & $2580 \pm 110$ & TF-783 & $2495 \pm 100$ & Agrawal et al., 1969 \\
\hline $\mathrm{Ba}-80$ & $4050 \pm 120$ & ТF-942 & $4055 \pm 110$ & Agrawal et al., 1971 \\
\hline \multirow[t]{5}{*}{ Ba- 85} & $4030 \pm 100$ & TF-56 & $3990 \pm 110$ & Kusumgar et al., 1963 \\
\hline & & SL-8 & $4020 \pm 100$ & Ellis and Sharp, 1964 \\
\hline & & LJ J 175 & 4080 & \\
\hline & & A-219 & $4240 \pm 150$ & Kusumgar et al., 1963 \\
\hline & & C-1 & $3979 \pm 350$ & \\
\hline
\end{tabular}

Comment (P.P.): the table shows satisfactory agreement with other laboratories.

II. ATMOSPHERIC $\mathrm{CO}_{2}$ SAMPLES

Data reported here are derived from atmospheric $\mathrm{CO}_{2}$ samples coll. at Jaslovské Bohunice and Bratislava. Measurements were made as part of continuing study of the $\mathrm{C}^{14}$ transport within the carbon reservoirs and as a part of our radioecological program.

\section{Jaslovské Bohunice series, 1968-1970}

Samples coll. at Jaslovské Bohunice ( $48^{\circ} 29^{\prime} \mathrm{N}$ Lat, $17^{\circ} 37^{\prime} \mathrm{E}$ Long), dist. Trnava, SW Slovakia, at ground level. The sampling sta. is far from any source of fossil fuel $\mathrm{CO}_{2}$. Sample of $\mathrm{CO}_{2}$ is coll. by bubbling air through $\mathrm{Ba}(\mathrm{OH})_{2}$ sampler.

TABle 2

Jaslovské Bohunice 1968-1970

\begin{tabular}{lll}
\hline Sample no. & \multicolumn{1}{c}{ Date } & $\delta \mathrm{C}^{14 \%} \%$ \\
\hline Ba-1 & Jan. 19, 31, 1968 & $63.6 \pm 3.0$ \\
Ba-2 & Feb. 14, 28, 1968 & $64.4 \pm 2.3$ \\
Ba-3 & April 30, 1968 & $72.5 \pm 1.0$ \\
Ba-4 & May 7, 1968 & $64.4 \pm 0.9$ \\
\hline
\end{tabular}


Table 2 (continued)

\begin{tabular}{|c|c|c|}
\hline Sample no. & Date & $\delta \mathrm{C}^{14 \%} \%$ \\
\hline $\mathrm{Ba}-5$ & May 22, 1968 & $81.0 \pm 3.3$ \\
\hline $\mathrm{Ba}-6$ & June 4, 1968 & $65.0 \pm 2.4$ \\
\hline $\mathrm{Ba}-7$ & June 19, 1968 & $65.7 \pm 1.9$ \\
\hline $\mathrm{Ba}-8$ & July 16, 1968 & $56.0 \pm 0.8$ \\
\hline Ва- 9 & Sept. 3, 1968 & $67.2 \pm 1.2$ \\
\hline $\mathrm{Ba}-10$ & Sept. 17, 1968 & $69.0 \pm 1.2$ \\
\hline $\mathrm{Ba}-11$ & Oct. 2, 1968 & $63.6 \pm 1.3$ \\
\hline Ba-12 & Oct. 22,1968 & $58.6 \pm 1.1$ \\
\hline $\mathrm{Ba}-13$ & Nov. 5, 1968 & $68.0 \pm 1.1$ \\
\hline Ba-14 & Jan. 7, 1969 & $50.2 \pm 2.4$ \\
\hline $\mathrm{Ba}-15$ & Feb. 18, 1969 & $43.2 \pm 2.0$ \\
\hline $\mathrm{Ba}-16$ & March 4, 1969 & $50.0 \pm 2.1$ \\
\hline $\mathrm{Ba}-17$ & March 18, 1969 & $62.6 \pm 2.2$ \\
\hline Ba-18 & April 2, 1969 & $66.7 \pm 2.4$ \\
\hline Ba-19 & April 15, 1969 & $64.2 \pm 2.4$ \\
\hline Ba-20 & May 13, 1969 & $74.2 \pm 2.2$ \\
\hline $\mathrm{Ba}-21$ & May 27, 1969 & $63.0 \pm 2.0$ \\
\hline Ba-22 & July 22, 1969 & $59.5 \pm 2.0$ \\
\hline Ba-23 & Sept. 10, 1969 & $56.3 \pm 2.4$ \\
\hline Ba-24 & Sept. 29, 1969 & $60.3 \pm 2.2$ \\
\hline $\mathrm{Ba}-49$ & Jan. 21, 1970 & $45.2 \pm 1.6$ \\
\hline Ba- 50 & Feb. 24, 1970 & $38.4 \pm 1.5$ \\
\hline Ва-46 & March 17,1970 & $43.5 \pm 1.5$ \\
\hline Вa-96 & April 7, 1970 & $51.3 \pm 1.4$ \\
\hline Ва-97 & April 30,1970 & $50.4 \pm 1.3$ \\
\hline Ba- 48 & May 5,1970 & $46.6 \pm 1.6$ \\
\hline $\mathrm{Ba}-45$ & June 12,1970 & $52.8 \pm 2.0$ \\
\hline Ba-98 & July 21,1970 & $53.1 \pm 1.4$ \\
\hline Ba-47 & Aug. 4, 1970 & $50.6 \pm 2.1$ \\
\hline Ba- 57 & Oct. 13,1970 & $51.4 \pm 2.0$ \\
\hline
\end{tabular}

Comment (P.P.): decrease in $\mathrm{C}^{14}$ concentrations during 1970 is noticeable.

\section{Bratislava series, 1969-1970}

Atmospheric $\mathrm{CO}_{2}$ is coll. weekly on the roof of the department building by static absorption of concentrated $\mathrm{NaOH}$ solution. The department is situated in the center of town.

TABLE 3

Bratislava 1969-1970

\begin{tabular}{llc}
\hline Sample no. & \multicolumn{1}{c}{ Date } & $\delta \mathrm{C}^{14} \%$ \\
\hline Ba-25 & Feb. 11-17, 1969 & $42.5 \pm 2.4$ \\
Ba-26 & March 3-10, 1969 & $55.7 \pm 2.4$ \\
\hline
\end{tabular}


Table 3 (continued)

\begin{tabular}{llc}
\hline Sample no. & \multicolumn{1}{c}{ Date } & $\delta \mathrm{C}^{140 \%}$ \\
\hline Ba-27 & April 28-May 5, 1969 & $63.2 \pm 2.4$ \\
Ba-95 & May 26-June 2, 1969 & $63.4 \pm 2.3$ \\
Ba-28 & July 7-14, 1969 & $58.4 \pm 2.3$ \\
Ba-29 & July 28-Aug. 11, 1969 & $52.3 \pm 2.1$ \\
Ba-92 & Oct. 26-Nov. 2, 1969 & $51.0 \pm 2.8$ \\
Ba-128 & Dec. 3-11, 1969 & $47.2 \pm 2.1$ \\
Ba-94 & Jan. 5-12, 1970 & $40.6 \pm 2.2$ \\
Ba-99 & Feb. 11-18, 1970 & $43.0 \pm 1.9$ \\
Ba-100 & March 4-11, 1970 & $41.7 \pm 2.1$ \\
Ba-108 & April 2-9, 1970 & $50.1 \pm 2.1$ \\
Ba-119 & May 12-19, 1970 & $47.9 \pm 1.9$ \\
Ba-109 & June 15-23, 1970 & $50.1 \pm 2.0$ \\
Ba-110 & July 16-24, 1970 & $55.3 \pm 2.0$ \\
Ba-133 & Aug. 12-21, 1970 & $51.2 \pm 1.2$ \\
Ba-120 & Sept. 8-17, 1970 & $48.5 \pm 1.9$ \\
Ba-134 & Oct. 7-16, 1970 & $49.2 \pm 1.2$ \\
Ba-126 & Nov. 12-20, 1970 & $51.3 \pm 2.0$ \\
Ba-127 & Dec. 8-16, 1970 & $42.5 \pm 1.8$ \\
&
\end{tabular}

Comment (P.P.): a clear industrial effect was observed in winter. $\mathrm{C}^{14}$ concentrations still show seasonal fluctuations with maximum in summer months. Results agree with present theories of $\mathbf{C}^{14}$ transport through carbon reservoirs (Povinec et al., 1971). Tables 2 and 3 show that daily and weekly concentrations of $\mathrm{C}^{14}$ in atmospheric $\mathrm{CO}_{2}$ are comparable.

III. BIOSPHERIC SAMPLES

Various biospheric materials were dated for radioecological purposes.

TABle 4

Modern plant samples

\begin{tabular}{lllc}
\hline Sample no. & \multicolumn{1}{c}{ Sample } & Date & $\delta \mathrm{C}^{14 \%}$ \\
\hline Ba-33 & Wheat straw & June 1966 & $75 \pm 3$ \\
Ba-32 & Nut twigs & Sept. 1968 & $63 \pm 3$ \\
Ba-34 & Sugar & Nov. 1968 & $73 \pm 3$ \\
Ba-53 & Grass & June 1970 & $56.8 \pm 2.1$ \\
Ba-52 & Wheat straw & July 1970 & $57.3 \pm 1.9$ \\
Ba-54 & Barley grain & July 1970 & $59.5 \pm 2.0$ \\
Ba-55 & Potatoes & Oct. 1970 & $52.7 \pm 2.0$ \\
Ba-56 & Apples & Oct. 1970 & $59.4 \pm 2.1$ \\
\hline
\end{tabular}




\section{Jurský šúr series}

IV. GEOLOGIC SAMPLES

Peat from bog Jurský šúr near Jur (48 $18^{\circ} 15^{\prime} \mathrm{N}$ Lat, $17^{\circ} 14^{\prime} \mathrm{E}$ Long), dist. Bratislava, SW Slovakia. Coll. and subm. 1968 by E. Povincová, Dept. Phys. Geog., Comenius Univ., Bratislava.

\section{Ba-39. Jurský šúr 1/68}

$4200 \pm 220$

Peat from depth 0.80 to $0.85 \mathrm{~m}$.

\section{Ba-40. Jurský šúr 2/68}

$12,000 \pm 260$

Peat from depth 2.65 to $2.70 \mathrm{~m}$.

\section{1,050 в.c.}

\section{Domica Cave series}

Stalagmite from Domica Cave $\left(48^{\circ} 30^{\prime} \mathrm{N}\right.$ Lat, $20^{\circ} 20^{\prime} \mathrm{E}$ Long) $10 \mathrm{~km}$ SE of Plešivec, dist. Rožnava, S Slovakia. Coll. and subm. 1970 by M. Liška, Slov. ústav pam. starost., Bratislava. Comment (P.P.): outer layer of sample etched off with dilute acid, inner part measured.

\section{Ba-106. Domica, No. 1}

$13,740 \pm 300$

Center part of stalagmite.

\section{2,790 B.C.}

\section{Ba-107. Domica, No. 2}

$8680 \pm 220$

Outer part of same stalagmite.

6730 B.C.

\section{Travertine series}

In collaboration with Geol. Inst. Slov. Acad. Sci., Bratislava we are studying the origin of travertine from different localities of Slovakia. Cioll. 1971 and subm. by R. Demovič. Comment (R.D.): $\delta C^{13}$ values were measured in Central Isotope Lab., Gottingen Univ., and are quoted relative to the PDB standard.

\section{Ba-114. Bešeňová}

$10,400 \pm 300$ 8450 в.C. $\delta C^{13}=+8.0 \%$

Travertine from sinter cascade near village Bešenová $\left(49^{\circ} 6^{\prime} \mathrm{N}\right.$ Lat, $19^{\circ} 25^{\prime}$ E Long), dist. Lipt. Mikuláš, N Slovakia. Yellow-brown, hard travertine. Comment (P.P.): no correction for isotopic fractionation.

\section{Ba-116. Vrútky-Dubná Skala}

Travertine from quarry opposite railway sta. Vrútky $\left(49^{\circ} 7^{\prime} \mathrm{N}\right.$ Lat, $18^{\circ} 54^{\prime} \mathrm{E}$ Long), dist. Martin, N Slovakia. Brown, very hard travertine. Comment (R.D.): travertine supposedly originated in Miocene. 


\section{Jazdiareň series}

\section{ARCHAEOLOGIC SAMPLES}

Wood from supporting beams from riding-ground building in Prague, Bohemia. Subm. 1968 by J. Lexa, Research Inst. Wood, Bratislava.

\section{Ba-37. Jazdiareň D 2}

Wood from Beam D-2.

\section{Ba-78. Jazdiareň D 4}

Wood from Beam D-4.

\section{Mirbach's Palace series}

Wooden beams from Mirbach's palace, Bratislava, Slovakia. Subm. 1968 by J. Lexa.

\section{Ba-30. Mirbach's Palace M 3}

Wood from Beam M-3.

\section{Ba-74. Mirbach's Palace M 2}

Wood from Beam M-2.

\section{Lužany series}

Samples from burial barrow discovered at Lužany $\left(48^{\circ} 31^{\prime} \mathrm{N}\right.$ Lat, $18^{\circ} 1^{\prime}$ E Long), dist. Topolčany, W Slovakia. Coll. 1967 and subm. by J. Paulík, Slov. Natl. Mus., Bratislava.

\section{Ba-38. Lužany 1}

$3040 \pm 160$

Charcoal from Tomb I/67/, top layer.

\section{Ba-90. Lužany 2}

$3260 \pm 110$

Charcoal from Tomb I/67/, bottom layer.

\section{Ba-73. Rudno}

A.D. 1420

Fragment of wooden beam from church at Rudno $\left(48^{\circ} 54^{\prime} \mathrm{N}\right.$ Lat, $18^{\circ} 45^{\prime}$ E Long), dist. Martin, N Slovakia. Coll. 1968 by P. Povinec.

\section{Ochodnica series}

During excavations for a new building, a destroyed wooden cellar was found $1.80 \mathrm{~m}$ below surface at Ochodnica $\left(49^{\circ} 23^{\prime} \mathrm{N}\right.$ Lat, $18^{\circ} 46^{\prime} \mathrm{E}$ Long), dist. Čadca, NW Slovakia. Coll. 1969 by P. Povinec.

Ba-82. Ochodnica, No. 1

Wood from a pale pit, Sec. A-1. 
Ba-83. Ochodnica, No. 2

Wood from a supporting beam, Sec. B-4.

Ba-84. Ochodnica, No. 3

Wood from a supporting beam, Sec. D-4.
90
A.D. 1960

$90 \pm 70$

A.D. 1875

\section{Plavecké Podhradie series}

Excavations made by Slov. Natl. Mus., Bratislava, uncovered a Celtic settlement with pale buildings on hill "Pohanská" $\left(48^{\circ} 29^{\prime} 20^{\prime \prime} \mathrm{N}\right.$ Lat, $17^{\circ} 16^{\prime} 20^{\prime \prime}$ E Long) near Plavecké Podhradie, dist. Senica, W Slovakia. Samples were charcoal fragments from construction parts of buildings. Coll. and subm. 1969 by J. Paulík.

Ba-88. Plavecké Podhradie, No. 1

$1980 \pm 90$

Charcoal from a pale pit, Sec. MO1-1 ABl 2 .

30 B.C.

Ba-89. Plavecké Podhradie, No. 2

$2050 \pm 90$

Charcoal from Beam 2, Sec. m-4-5 ABC.

100 B.C.

\section{Ba-101. Smolenice}

Carbonized grain from early Hallstatt age site on "Molpír" hill near Smolenice (48 $30^{\prime} \mathrm{N}$ Lat, $17^{\circ} 25^{\prime}$ E Long), dist. Trnava, SW Slovakia. Sample from nearly destroyed store vessel from burnt hut. Coll. 1967 by M. Dušek, subm. by E. Hajnalová, Archaeol. Inst. Slovak Acad. Sci., Nitra.

\section{Ba-102. Nová Lesná}

$2000 \pm 150$

Carbonized tree trunk from part of wooden fortification in sand mine

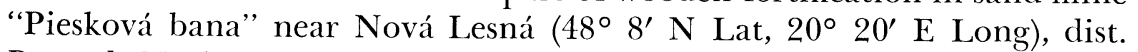
Poprad, N Slovakia. Sample from Object 1, Sec. 3-K, depth $45 \mathrm{~cm}$. Coll. Aug. 1971 by L. Veliačik; subm. by E. Hajnalová.

\section{Ba-103. Vráble}

$3310 \pm 190$

Carbonized grain from late Bronze age village on loess eminence "Fidvár" on left bank of Zitava R. near Vráble $\left(48^{\circ} 15^{\prime} \mathrm{N}\right.$ Lat, $18^{\circ} 19^{\prime} \mathrm{E}$ Long), dist. Nitra, S Slovakia. Sample was from destroyed hut. Coll. Aug. 1967 by K. Sedlák; subm. by E. Hajnalová.

\section{Ducové series}

Charcoal from $\log$ which was a part of fortification on calcite tongue “Kostelec," $80 \mathrm{~m}$ above Váh R. inundation area near Ducové $\left(48^{\circ} 58^{\prime} \mathrm{N}\right.$ Lat, $17^{\circ} 51^{\prime}$ E Long), dist. Trnava, SW Slovakia. Bottom layer is dolomite calcite, above 40 to $80 \mathrm{~cm}$ humus soil mixed with organic remains. Samples from 40 to $45 \mathrm{~cm}$ depth of humus clay mixed with calcite gravel. Coll. 1970 by A. Ruttkay; subm. by E. Hajnalová. 
Ba-104. Ducové, No. 1

Charcoal from beam between $\mathrm{W}$ middle rooms.

\section{Ba-121. Ducové, No 2}

Charcoal from beam of $\mathrm{W}$ wall of $\mathrm{W}$ room.

Ba-129. Ducové, No. 3

Charcoal from beam of $\mathrm{E}$ wall.

\section{Ba-130. Ducové, No. 4}

Charcoal from beam between middle and $\mathrm{E}$ walls.

\section{Ba-105. Liptovská Mara}

Charcoal (Fogus) from cultural layer in valley of Váh R. near Liptovská Mara $\left(49^{\circ} 7^{\prime} \mathrm{N}\right.$ Lat, $19^{\circ} 29^{\prime} \mathrm{E}$ Long), dist. Lipt. Mikuláš, $\mathrm{N}$ Slovakia. Sample from Sec. I/68, depth 75 to $80 \mathrm{~cm}$. Coll. July, 1968 by K. Pieta; subm. by E. Hajnalová.

\section{REFERENCES}

Agrawal, D. P., Gupta, S. K., and Kusumgar, Shcela, 1969, Tata Institute radiocarbon date list VII: Radiocarbon, v. 11, p. 502-508.

1971. Tata Institute radiocarbon date list VIII: Radiocarbon, v. 13, p. 84-93. Broecker, W. S. and Olson, E. A., 1961, Lamont natural radiocarbon measurements VIII: Radiocarbon, v. 3, p. 176-204.

Ellis, J. G. and Sharp R. A., 1964, Sharp Laboratories measurements I: Radiocarbon, v. 6, p. 108-109.

Kusumgar, Sheela, Lal D., and Sarna, R. P., 1963, Tata Institute radiocarbon date list I: Radiocarbon, v. 5, p. 273-282.

Lal, Devendra, 1965, Single-stage high-yield hydrogenation of $\mathrm{CO}_{2}$ to methane using the hydrogen of water: 6 th internatl. conf. radiocarbon and tritium dating Proc., Pullman, Washington, June 7-11, 1965, p. 487-490.

Povinec, Pavol, 1972, Preparation of methane gas filling for proportional $\mathbf{H}^{3}$ and $\mathbf{C}^{1 / 4}$ counters: Radiochem. Radioanal. Letters, v. 9, p. 127-135.

Povinec, P., Chudy, M., and Sáró, S., 1971, Vyvoj metód stanovenia velmi nízkych aktivít $\mathrm{C}^{14}$ proporcionálnym pocítacom: KJF UK, 15/71, Comenius Univ., Bratislava, 54 p.

Povinec, P., Chudy, M., and Seliga, M., 1971, Equipment for the absolute age determination using carbon isotope $\mathrm{C}^{14}$ : Cs. cas. fys., v. 21, p. 17-25. Physica, v, 11, p. $91-100$

Povinec, P., Sáró, S., Chudy, M., and Seliga, M., 1968, The rapid method of carbon-14 counting in atmospheric carbon dioxide: Internatl. Jour. Appl. Rad. Isotopes, v. 19 , p. $877-881$ 